\title{
Synthesizing a Cellulase like Chimeric Protein by Recombinant Molecular Biology Techniques
}

Hirendra Nath Banerjee*, Christopher Krauss, Valerie Smith, Kelly Mahaffey and Ava Boston

Department of Natural Pharmacy and Health Sciences, Elizabeth City State University, University of North Carolina, Elizabeth City, NC-27909, USA

\begin{abstract}
In order to meet the Renewable Fuels Standard demands for 30 billion gallons of biofuels by the end of 2020 , new technologies for generation of cellulosic ethanol must be exploited. Breaking down cellulose by cellulase enzyme is very important for this purpose but this is not thermostable and degrades at higher temperatures in bioreactors. Towards creation of a more ecologically friendly method of rendering bioethanol from cellulosic waste, we attempted to produce recombinant higher temperature resistant cellulases for use in bioreactors. The project involved molecular cloning of genes for cellulose-degrading enzymes based on bacterial source, expressing the recombinant proteins in $E$. coli and optimizing enzymatic activity. We were able to generate in vitro bacterial expression systems to produce recombinant His-tag purified protein which showed cellulase like activity.
\end{abstract}

\section{Introduction}

Cheap, clean, green energy production is a goal of Department of energy and EPA. Biofuels are made by converting renewable materials-for example, corn kernels, wood chips left over from pulp and paper production, prairie grasses, and even garbage--into fuels and chemicals. Most biofuels used today are made from the fermentation of starch from corn kernels. That process, although simple, is costly because of the high price of the corn kernels themselves.

Agricultural waste, such as corn stover (the leaves, stalks, and stripped cobs of corn plants, left over after harvest), is cheap. These materials are largely composed of cellulose, the chief component of plant-cell walls. Cellulose is far tougher to break down than starch. An additional complication is that while the fermentation reaction that breaks down corn starch needs just one enzyme, the degradation of cellulose requires a whole suite of enzymes, or cellulases, working in concert.

The cellulases currently used industrially, all of which were isolated from various species of plant-decaying filamentous fungi, are both slow and unstable, and, as a result, the process remains prohibitively expensive. Even a two-fold reduction in their cost could make a big difference to the economics of renewable fuels and chemicals; Thermostability is a requirement of efficient cellulases, because at higher temperatures, 70 or even 80 degrees Celsius--chemical reactions are more rapid. In addition, cellulose swells at higher temperatures, which makes it easier to break down. Unfortunately, the known cellulases from nature typically won't function at temperatures higher than about $50^{\circ} \mathrm{C}$. Cellulolytic anaerobic bacteria use macromolecular structures known as cellulosomes to hydrolyze recalcitrant cellulosic substrates [1,2]. Within the cellulosome, cellulases and other glycoside hydrolases $[3,4]$ are assembled onto multidomain scaffoldin proteins for efficient degradation of cellulosic substrates [4]. Cellulosome assembly is achieved by binding dockerin domains from enzymes with cohesin domains in scaffoldin, while localization with substrate is mediated by one or more Carbohydrate Binding Modules (CBMs) on the scaffoldin $[1,2,5]$. The modularity of cellulosomes has spurred interest in 'designer cellulosomes' [6], where different cellulases are synthetically combined for a specific application. Within a given glycoside hydrolase family, a diverse pool of potential cellulases would be beneficial for designer cellulosomes by providing a suite of enzymes with differing properties and an extensive platform for further enzyme engineering. Family 48 cellulases (Cel48) are ideal candidates for designer cellulosomes [3]. As one of the most important families of bacterial cellulases, they are usually a major constituent of bacterial cellulosomes [4,7-12]. Of the 116 bacterial Cel48 genes currently predicted in the CAZy database (http://www.cazy.org/) only 13 have been characterized. We chose SCHEMA recombination to plan to synthesize a diverse set of new family 48 sequences. SCHEMA is a structure-guided, site-directed protein recombination method that has been used to generate thousands of novel P450s, $\beta$-lactamases, and fungal cellulases. The chimeric proteins that are made by recombining natural sequences differ. Our objective for this project was to construct chimeric synthetic cellulase genes for production of thermostable cellulases for efficient breakdown of cellulose at high temperature.

\section{Materials and Methods}

Genomic DNA from bacteria Cellulomonas sp. (ATCC' 21399) was used as a template to do PCR using standard PCR reagents and assay conditions using the primers:

\begin{tabular}{|l|c|}
\hline CCELcdCTHEdock+Xbalfwd & $\begin{array}{c}\text { GCAATACTCTTCCCAGATTCTAGAATGACAT } \\
\text { ATAAAGTACCTGGTACTCCTTCTACT }\end{array}$ \\
\hline CCELcdCTHEdock+Xbalrev & $\begin{array}{c}\text { AGGTACTTTATATGTCATTCTAGAATCTGGG } \\
\text { AAGAGTATTGCATAAACTCCATTTGC }\end{array}$ \\
\hline
\end{tabular}

The amplicon was further sequenced and the obtained sequence (Figure 1) was subjected to NCBI-BLAST search and showed homology to A. thermophillum celA gene (Figure 2).

The amplicon was then cloned into a Gateway System (Invitrogen, USA) his-tag expression vector and BL-21 E. coli bacteria was transformed with this construct. The bacteria was then grown in LB medium and IPTG was used to induce the protein, which was then his-

*Corresponding author: Hirendra Nath Banerjee, Professor, Department of Natural Pharmacy and Health Sciences, Elizabeth City State University, University of North Carolina, Elizabeth City, NC-27909, USA, Tel: 2523353241; Fax: 2523353697; E-mail: bhirendranath@ecsu.edu

Received March 18, 2016; Accepted June 25, 2016; Published June 30, 2016

Citation: Banerjee HN, Krauss C, Smith V, Mahaffey K, Boston A (2016) Synthesizing a Cellulase like Chimeric Protein by Recombinant Molecular Biology Techniques. J Bioprocess Biotech 6: 285. doi:10.4172/2155-9821.1000285

Copyright: (c) 2016 Banerjee HN, et al. This is an open-access article distributed under the terms of the Creative Commons Attribution License, which permits unrestricted use, distribution, and reproduction in any medium, provided the original author and source are credited. 
CATATGGCCAGCAGCGATGATCCGTATAAGCAACGTTTCTTGGAACTGTGGGAAGA GTTGCACGATCCGAGCAACGGTTATTTCAGCTCCCATGGTATTCCGTACCACGCGGT CGAGACGCTGATCGTTGAGGCACCTGATTATGGCCACCTGACCACCAGCGAAGCGA TGTCTTACTATCTGTGGCTGGAAGCGCTGTACGGCAAATTTACGGGTGATTTTAGCT ATTTCATGAAGGCCTGGGAAACCATTGAGAAGTACATGATTCCGACCGAGCAGGAT CAACCGAACCGCTCCATGGCTGGTTACAATCCGGCTAAACCAGCGACCTATGCCCCT GAATGGGAAGAACCGAGCATGTATCCGTCTCAGCTGGACTTCAGCGCACCGGTGGG CATTGACCCGATTTACAATGAGCTGGTGTCCACCTATGGTACCAATACGATTTACGG TATGCACTGGCTGCTGGATGTGGATAACTGGTACGGCTTTGGCCGTCGTGCGGACCG TATCAGCAGCCCAGCCTATATCAACACCTTCCAACGTGGCAGCCAAGAGTCCGTGTG GGAGACGATCCCGCAACCGTGCTGGGATGATCTGACCATCGGTGGCCGTAACGGTTT TCTGGACCTGTTTGTCGGCGATAGCCAGTACTCGGCACAATTTAAGTACACGAATGC ACCGGACGCGGATGCGCGTGCCATCCAGGCGACGTACTGGGCGAACCAGTGGGCGA AAGAGCACGGCGTGAATTTGAGCCAGTATGTTAAGAAGGCAAGCCGCATGGGCGAC TACCTGCGCTATGCAATGTTCGACAAATACTTTCGTAAAATTGGTGATTCCAAACAA GCAGGTACCGGCTACGACGCAGCCCATTACCTGCTGTCCTGGTACTATGCGTGGGGT GGTGGCATCACGGCTGATTGGGCATGGATTATTGGCTGTTCCCACGTTCATGCAGGC TACCAGAATCCGATGACGGCGTGGATTCTGGCCAACGATCCGGAGTTTAAACCGGA AAGCCCGAACGGTGCTAATGATTGGGCGAAAAGCCTGGAGCGCCAGCTGGAGTTCT ATCAATGGCTGCAGAGCGCTGAGGGTGCAATCGCAGGTGGTGCGACGAATAGCTAC AAAGGTCGCTACGAAACCCTGCCAGCAGGTATCAGCACGTTCTATGGCATGGCGTAT GAAGAACATCCGGTGTACCTGGATCCGGGTAGCAACACGTGGTTTGGCTTTCAGGCG TGGACGATGCAGCGCGTGGCGGAATACTACTATCTGACCGGTGATACGCGTGCAGA GCAACTGTTGGACAAATGGGTCGATTGGATCAAGTCCGTTGTTCGTCTGAACAGCGA CGGCACCTTCGAGATTCCGGGTAACCTGGAGTGGTCGGGTCAACCGGACACCTGGA CCGGTACTTACACGGGTAATCCGAACCTGCATGTCAGCGTTGTTTCTTATCGTACGG ACTTGGGTGCAGCGGGTTCTCTGGCAAATGCTCTGCTGTACTATGCCAAAACCAGCG GTGACGACGAAGCACGTAATCTGGCGAAAGAATTGCTGGACCGTATGTGGAACCTG TACCGTGACGACAAAGGTTTGTCCGCACCGGAGACTCGCGAAGATTACGTCCGCTTT TTCGAACAAGAGGTTTACGTTCCACAGGGTTGGTCTGGTACGATGCCTAACGGCGAT CGTATCGAACCGGGTGTTACTTTCCTGGACATCCGCTCGAAATACCTGAACGACCCG 


\begin{tabular}{|c|c|c|}
\hline Query & 73 & AACGGTTATTTCAGCTCCCATGGTATTCCGTACCACGCGGTCGAGACGCTGATCGTTGAG 132 \\
\hline & & 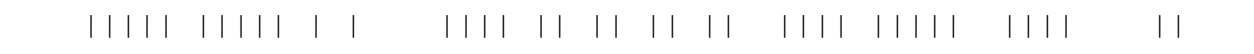 \\
\hline Sbjet & 3304 & AACGGGTATTTTAACCAGGATGGGATACCATATCATTCGGTAGAGACATTGATATGCGAA \\
\hline Query & 133 & GCACCTGATTATGGCCACCTGACCACCAGCGAAGCGATGTCTTACTATCTGTGGCTGGAA 192 \\
\hline & & 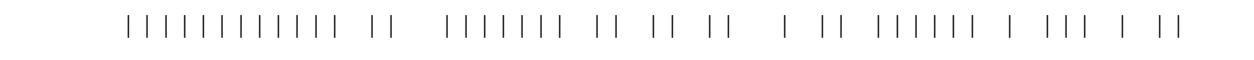 \\
\hline Sbjet & 3364 & CGACCTGATTATGGTCATTTGACCACGAGTGAGGCATTTTCGTACTATGTATGGTTAGAG 3423 \\
\hline Query & 193 & GCGCTGTACGGCAAATTTACGGGTGATTTTAGCTATTTCATGAAGGCCTGGGAAACCATT 252 \\
\hline & & 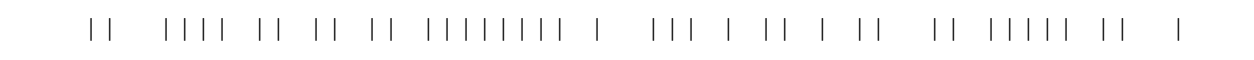 \\
\hline Sbjet & 3424 & GCAGTGTATGGTAAGTTAACGGGTGACTGGAGCAAATTTAAGACAGCATGGGACACATTA 3483 \\
\hline Query & 253 & GAGAAGTACATGATTCCGACCGAGCAGGATCAACCGAACCGCTCCATGGCTGGTTACAAT 312 \\
\hline & & $|1||1||||||||||||||||||||||||||| \mid$ \\
\hline Sbjet & 3484 & GAGAAGTATATGATACCATCAGCGGAAGATCAGCCGATGAGGTCA---------TATGAT 3534 \\
\hline Query & 313 & CCGGCTAAACCAGCGACCTATGCCCCTGAATGGGAAGAACCGAGCATGTATCCGTCTCAG 372 \\
\hline & & 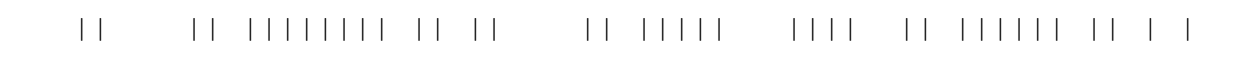 \\
\hline Sbjet & 3535 & CCTAACAAGCCAGCGACATACGCAGGGGAGTGGGAGACACCGGACAAGTATCCATCGCCG \\
\hline Query & 373 & CTGGACTTCAGCGCACCGGTGGGCATTGACCCGATTTACAATGAGCTGGTGTCCACCTAT 432 \\
\hline & & 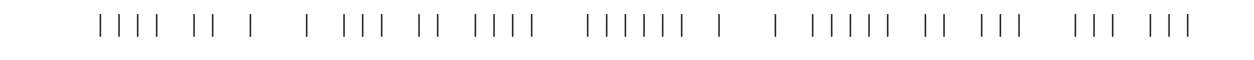 \\
\hline Sbjet & 3595 & TTGGAGTTTAATGTACCTGTTGGCAAAGACCCGTTGCATAATGAACTTGTGAGCACATAT \\
\hline Query & 433 & GGTACCAATACGATTTACGGTATGCACTGGCTGCTGGATGTGGATAACTGGTACGGCTTT 492 \\
\hline & & ||||||$\quad||||||||||||||||||||||||||||||||||||||$ \\
\hline Sbjet & 3655 & GGTAGCACATTAATGTATGGTATGCACTGGTTGATGGACGTAGACAACTGGTATGGATAT \\
\hline Query & 493 & GGCCGTCGTGCGGACCGTATCAGCAGCCCAGCCTATATCAACACCTTCCAACGTGGCAGC 552 \\
\hline & & 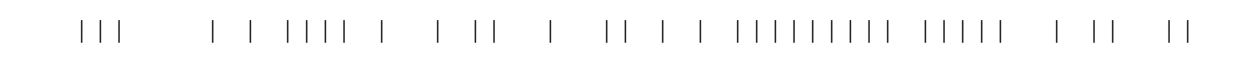 \\
\hline Sbjet & 3715 & GGCAAGAGAGGGGACGGAGTAAGTCGGGCATCATTTATCAACACGTTCCAGAGAGG--GC 3772 \\
\hline Query & 553 & C--AAGAGTCCGTGTGGGAGACGATCCCGCAACCGTGCTGGGATGATCTGACCATCGGTG 610 \\
\hline & & $|\quad||||||||||||||||||||||||||||||||||||| \mid$ \\
\hline Sbjet & 3773 & CTGAGGAGTCTGTATGGGAGACGGTGCCGCATCCGAGCTGGGAGGAATTCAAGTGGGGCG 3832 \\
\hline Query & 611 & GCCGTAACGGTTTTCTGGACCTGTTTGTCGGCGATAGCCAGTACTCGGCACAATTTAAGT $\quad 670$ \\
\hline & & 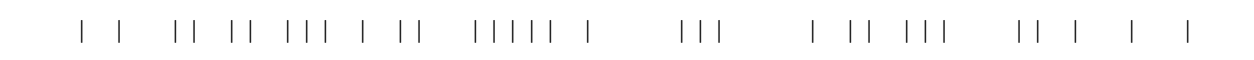 \\
\hline
\end{tabular}




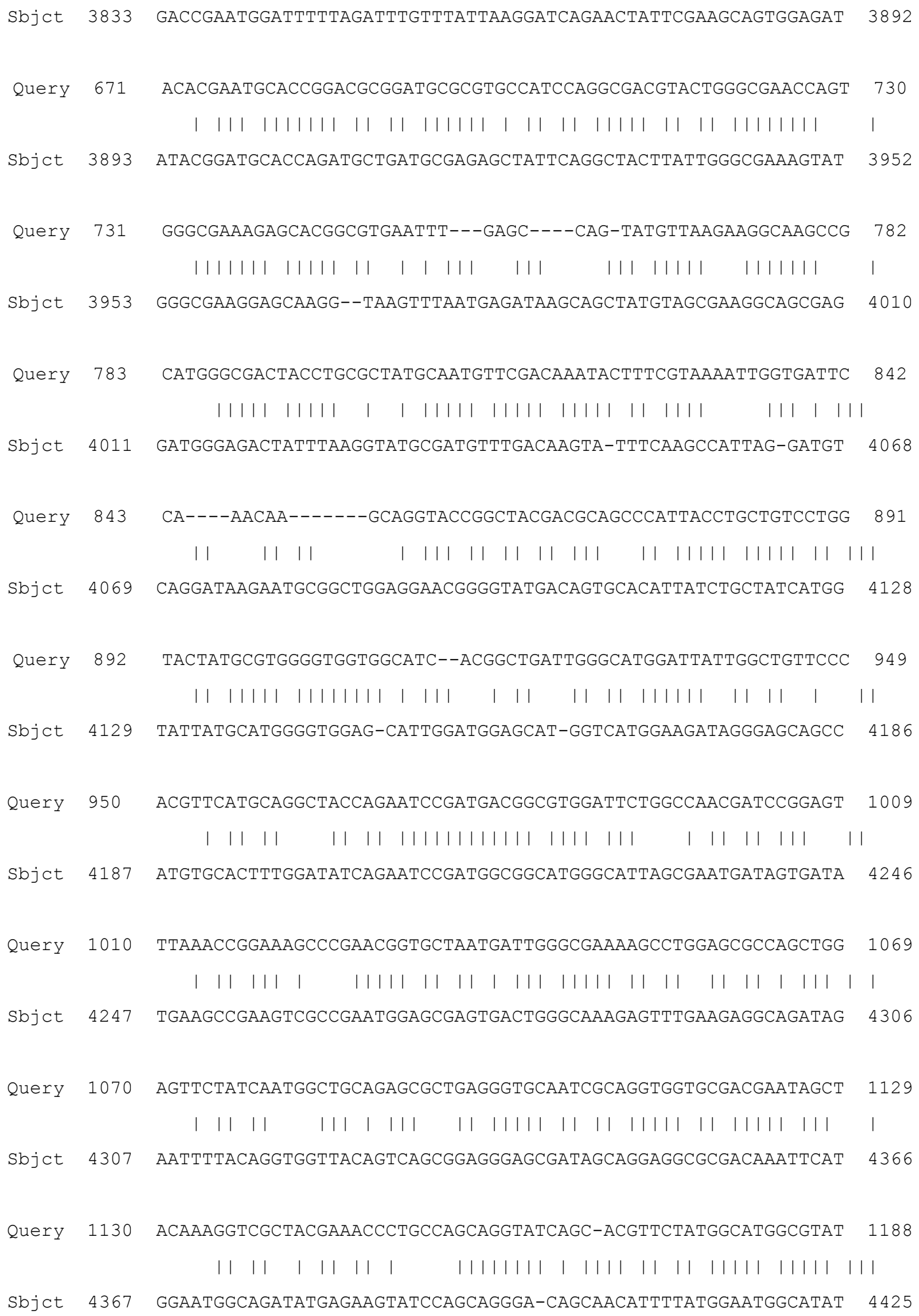




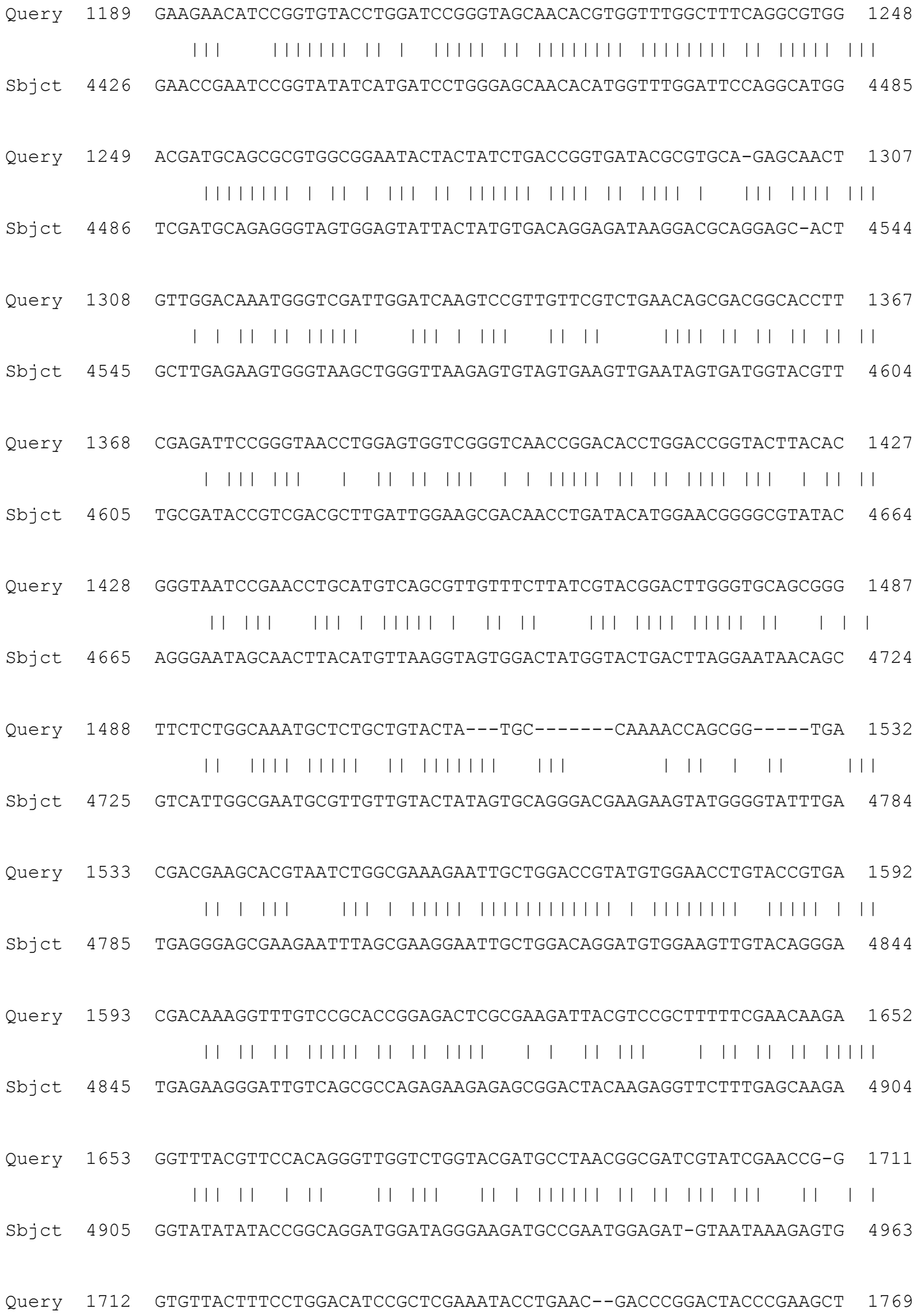


Citation: Banerjee HN, Krauss C, Smith V, Mahaffey K, Boston A (2016) Synthesizing a Cellulase like Chimeric Protein by Recombinant Molecular Biology Techniques. J Bioprocess Biotech 6: 285. doi:10.4172/2155-9821.1000285

\begin{tabular}{|c|c|c|c|}
\hline Sbjet & 4964 & GAGTTAAGTTTATAGACATAAGGAGCAAGTA--TAAACAAGATCCTGATTGGCCGAAGTT & 5021 \\
\hline \multirow[t]{2}{*}{ Query } & 1770 & GCAGCAGGCGTATAACGAAGGCAAAGCGCCAGTGTTCAACTATCACCGTTTCTGGGCTCA & 1829 \\
\hline & & 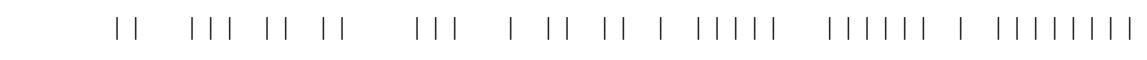 & $\mid 1$ \\
\hline \multirow[t]{3}{*}{ Sbjet } & 5022 & AGAGGCGGCATACAAGTCAGGGCAGGCACCTGAGTTCAGATATCACAGGTTCTGGGCACA & 5081 \\
\hline & Query & 1830 AтGCGACATCGCTATCGCGAACGGCTTGTATAGCAтTCTGTTTGGCA 1876 & \\
\hline & ct & $\begin{array}{r}|||||||||||||||||||||||c||||||||||||||||||| \\
5082 \text { GTGCGACATAGCAATAGCTAATGCAACATATGAAATACTGTTTGGCA } 5128\end{array}$ & \\
\hline
\end{tabular}

A. thermophilum celA gene and manA pseudogene

Sequence ID: emb|Z86105.1| Length: 5513 Number of Matches: 1

Related Information

Range 1: 3304 to 5128 GenBankGraphics Next Match Previous Match

\begin{tabular}{|l|l|l|l|l|}
\hline \multicolumn{5}{|c|}{ Alignment statistics for match \#1 } \\
\hline Score & Expect & Identities & Gaps & Strand \\
\hline 369 bits (408) & $2 \mathrm{e}-97$ & $1202 / 1847(65 \%)$ & $65 / 1847(3 \%)$ & Plus/Plus \\
\hline
\end{tabular}

Figure 2: NCBI-BLAST search result of the sequenced amplicon DNA.

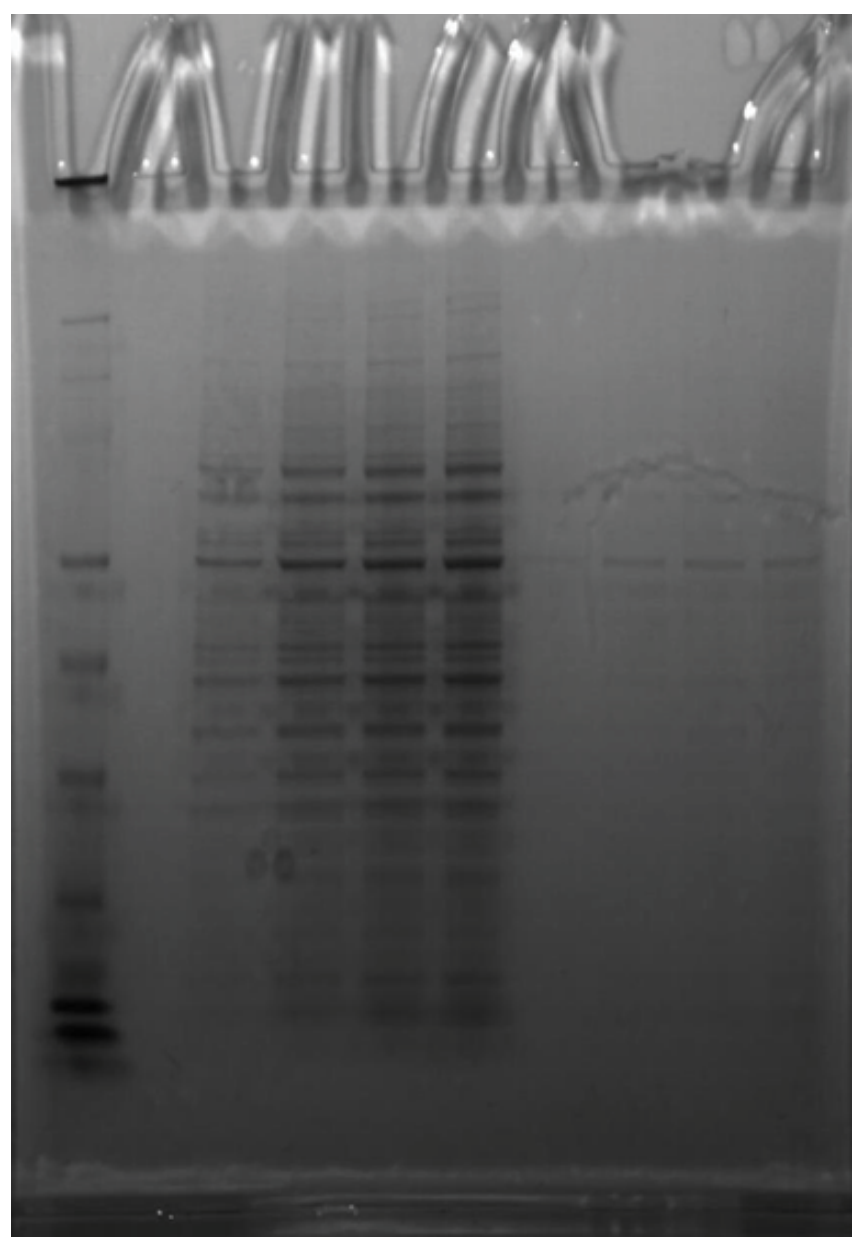

Figure 3: Lane 1=Protein marker, Lane 3-6=Different fractions of bacterial protein expressed, Lane 7-10=His-tag purified recombinant cellulase like Chimeric protein. 
Citation: Banerjee HN, Krauss C, Smith V, Mahaffey K, Boston A (2016) Synthesizing a Cellulase like Chimeric Protein by Recombinant Molecular Biology Techniques. J Bioprocess Biotech 6: 285. doi:10.4172/2155-9821.1000285

\begin{tabular}{|c|c|}
\hline Enzyme Concentration & Bioactivity \\
\hline $100 \mu \mathrm{g} / \mu \mathrm{l}$ & 0.50 \\
\hline $50 \mu \mathrm{g} / \mu \mathrm{l}$ & 0.25 \\
\hline $25 \mu \mathrm{g} / \mu \mathrm{l}$ & 0.15 \\
\hline $10 \mu \mathrm{g} / \mu \mathrm{l}$ & 0.05 \\
\hline
\end{tabular}

Table 1: Showing cellulase bioactivity of the novel recombinant chimeric protein by Park Johnson Assay.

tag purified using a nickel column (please see the gel picture in Figure 3 ), protein concentration was measured by using standard Bradford method (Sigma, USA).

\section{Cellulase Assay}

\section{Method}

A standard assay for cellulase activity was performed with a reaction mixture containing $0.52 \%$ carboxymethyl cellulose in $10 \mathrm{mM}$ sodium phosphate $(\mathrm{pH} 7.0)$ at $30^{\circ} \mathrm{C}$. Reduced sugar produced by the reaction was determined using the method described by Park and Johnson [13] using a standard BioRad (USA) spectrophotometer.

\section{Results and Discussion}

We were interested to synthesize a chimeric synthetic cellulase gene from the different cellulases DNA sequence that are there in the gene bank to produce a thermostable cellulose, our initial bioinformatics analysis by using the CAZy database and SCHEMA recombination to design gene sequences which will fulfill those conditions resulted in production of a chimeric protein. We derived the following full length DNA sequence (Figure 1) which showed homology to Cel A gene of A. thermophillum (Figure 2) and we expressed and purified the recombinant protein by His-tag method (Figure 3). The activity of this novel chimeric protein was determined to be cellulase when tested for activity by standard Park Johnson assay (Table 1). Thus our recombinant chimeric proteins have definite Cellulase enzyme characteristics. We look forward to scaling up productions and temperature and $\mathrm{pH}$ stability testing for its usefulness for bioremediation.

\section{Acknowledgements}

Supported by USA - Department of Energy (DOE) Grant and NIH Grant \# T34GM100831 to Dr. Hirendra Nath Banerjee.

\section{References}

1. Cheng YS, Ko TP, Wu TH, Ma Y, Huang CH, et al. (2010) Crystal structure and substrate-binding mode of cellulase 12A from Thermotoga maritima. Proteins 79: 1193-1204.

2. Blumer-Schuette SE, Kataeva I, Westpheling J, Adams MW, Kelly RM (2008) Extremely thermophilic microorganisms for biomass conversion: status and prospects. Curr Opin Biotechnol 19: 210-217.

3. Fierobe HP, Bayer EA, Tardif C, Czjzek M, Mechaly A, et al. (2002) Degradation of cellulose substrates by cellulosome chimeras. Substrate targeting versus proximity of enzyme components. J Biol Chem 277: 49621-49630.

4. Blum DL, Kataeva IA, Li XL, Ljungdahl LG (2000) Feruloyl esterase activity of the Clostridium thermocellum cellulosome can be attributed to previously unknown domains of XynY and XynZ. J Bacteriol 182: 1346-1351.

5. Lynd LR, Weimer PJ, van Zyl WH, Pretorius IS (2002) Microbial cellulose utilization: fundamentals and biotechnology. Microbiol Mol Biol Rev 66: 506577.

6. Boraston AB, Bolam DN, Gilbert HJ, Davies GJ (2004) Carbohydrate-binding modules: fine-tuning polysaccharide recognition. Biochem J 382: 769-781.

7. Tamaru Y, Doi RH (2001) Pectate lyase A, an enzymatic subunit of the Clostridium cellulovorans cellulosome. Proc Natl Acad Sci USA 98: 4125-4129.

8. Cantarel BL, Coutinho PM, Rancurel C, Bernard T, Lombard V, et al. (2009) The Carbohydrate-Active EnZymes database (CAZy): an expert resource for Glycogenomics. Nucleic Acids Res 37: 233-238.

9. Reverbel-Leroy C, Pages S, Belaich A, Belaich JP, Tardif C (1997) The processive endocellulase CelF, a major component of the Clostridium cellulolyticum cellulosome: purification and characterization of the recombinant form. J Bacteriol 179: 46-52.

10. Bronnenmeier K, Kundt K, Riedel K, Schwarz WH, Staudenbauer WL (1997) Structure of the Clostridium stercorarium gene celY encoding the exo-1,4-betaglucanase Avicelase II. Microbiology 143: 891-898

11. Wang WK, Kruus K, Wu JH (1993) Cloning and DNA sequence of the gene coding for Clostridium thermocellum cellulase Ss (CelS), a major cellulosome component. J Bacteriol 175: 1293-1302.

12. Vazana $Y$, Moraïs S, Barak $Y$, Lamed R, Bayer EA (2010) Interplay between Clostridium thermocellum family 48 and family 9 cellulases in cellulosomal versus noncellulosomal states. Appl Environ Microbiol 76: 3236-3243.

13. Park J, Johnson MJ (1949) A submicrodetermination of glucose. J Biol Chem 181: 149-151.
Citation: Banerjee HN, Krauss C, Smith V, Mahaffey K, Boston A (2016) Synthesizing a Cellulase like Chimeric Protein by Recombinant Molecula Biology Techniques. J Bioprocess Biotech 6: 285. doi:10.4172/2155 9821.1000285
OMICS International: Publication Benefits \& Features Unique features:

Increased global visibility of articles through worldwide distribution and indexing

Showcasing recent research output in a timely and updated manner

Special issues on the current trends of scientific research

Special features:

$700+$ Open Access Journa

$50,000+$ editorial team

Rapid review proces

Quality and quick editorial, review and publication processing

Indexing at major indexing services

Sharing Option: Social Networking Enabled

Authors, Reviewers and Editors rewarded with online Scientific Credits

Better discount for your subsequent articles

Submit your manuscript at: http://www.omicsonline.org/submission 